ITP-UH-22/13

\title{
On the Gribov problem in Yang-Mills theory
}

\author{
Olaf Lechtenfeld \\ Institut für Theoretische Physik and Riemann Center for Geometry and Physics, \\ Leibniz Universität Hannover, Appelstrasse 2, 30167 Hannover, Germany \\ URL: www.itp.uni-hannover.de/ lechtenf
}

\begin{abstract}
I briefly review the Gribov ambiguity of Yang-Mills theory, some of its features and attempts to control it, in particular the Gribov-Zwanziger proposal to restrict the functional integration in the Landau gauge to the Gribov region. This proposal is extended to an arbitrary gauge in such a way as to guarantee BRST invariance. The key insight is that any gauge change in the generating functional can be effected by a suitable field-dependent BRST transformation. I derive a simple analytic formula for the Jacobian of such a transformation, which yields an explicit recipe for the required transformation-parameter functional and allows for the computation of the Gribov horizon functional in any gauge, as I illustrate for the class of $R_{\xi}$ gauges.
\end{abstract}

Talk presented at SQS-13 during 29 July - 03 August, 2013, at JINR, Dubna, Russia 


\section{What is the Gribov ambiguity?}

Gauge theories are systems with redundant field variables. The simplest prototype is electrodynamics in $d$ spacetime dimensions, described by gauge potentials $A(x)=A_{\mu}(x) \mathrm{d} x^{\mu}$ with $\mu=0,1, \ldots, d-1$, subject to gauge transformations

$$
A \mapsto{ }^{U} A=U(\mathrm{~d}+A) U^{\dagger} \quad \text { with } \quad U(x)=\mathrm{e}^{\mathrm{i} \xi(x)}
$$

The configurations gauge equivalent to a given $A$ form the gauge orbit $\mathcal{O}_{A}=\left\{A^{\prime} \mid \exists U: A^{\prime}={ }^{U} A\right\}$, and the physical configuration space $\mathcal{P}$ is the space of gauge orbits or, equivalently, the quotient space $\mathcal{P}=\{A\} /\{U\}$, which is a topologically and geometrically complicated infinite-dimensional orbifold. The gauge redundancy is already relevant perturbatively, for the kinetic operator in the action possesses zero modes,

$$
\left(S^{(2)}\right)_{\mu \nu} \partial^{\nu} f \equiv\left(\eta_{\mu \nu} \square-\partial_{\mu} \partial_{\nu}\right) \partial^{\nu} f=0
$$

hence $S^{(2)}$ is not invertible on $\{A\}$. To proceed computationally, one needs to fix a gauge, which is a prescription of picking a representative $A$ from each gauge orbit, uniquely and completely, by a (local) 'ideal' condition $\chi(A, x)=0$. This is implemented in the generating-functional path integral $Z(J)$ via the Faddeev-Popov trick: Insert $1=\int \mathcal{D} U \delta\left(\chi\left({ }^{U} A\right)\right)|\operatorname{det} K(A)|$ with $K(A)=\left.\frac{\delta\left(\chi\left({ }^{U} A\right)\right)}{\delta U}\right|_{\chi=0}$, factor off the gauge-group volume and obtain

$$
\begin{aligned}
Z(J) & =\int \mathcal{D} A \delta(\chi(A))|\operatorname{det} K(A)| \mathrm{e}^{\frac{\mathrm{i}}{\hbar}\left[S_{0}(A)+\int J A\right]} \\
& =\int \mathcal{D}(A, C, \bar{C}, B) \mathrm{e}^{\frac{\mathrm{i}}{\hbar}\left[S_{0}(A)+\int \bar{C} K C+\int \chi(A) B+\int J A\right]} .
\end{aligned}
$$

Let us illustrate potential pitfalls in a two-dimensional toy example [1], with 'gauge fields' $(x)=(r, \theta) \in \mathbb{R}^{2}$ subject to 'gauge transformations' $\theta \mapsto \theta+\phi$. The gauge-fixing function $\chi$ might vanish for more than one angle $\theta_{i}=\theta_{i}(r)$, so in general

$$
\int \mathrm{d} \phi \delta(\chi(r, \theta+\phi))=\sum_{i}\left|\frac{\partial \chi}{\partial \theta}\left(r, \theta_{i}(r)\right)\right|^{-1} \quad \text { with } \quad \chi\left(r, \theta_{i}(r)\right)=0 .
$$

If the 'action function' $S=S(r)$ is independent of $\theta$ ('gauge invariance'), then

$$
Z=\int \mathrm{d}^{2} x \mathrm{e}^{\mathrm{i} S(r)}=\underbrace{\int \mathrm{d} \phi}_{2 \pi} \int \mathrm{d}^{2} x\left[\sum_{i}\left|\frac{\partial \chi}{\partial \theta}\left(r, \theta_{i}(r)\right)\right|^{-1}\right]^{-1} \underbrace{\delta(\chi(x))}_{\text {kills } \int \mathrm{d} \theta} \mathrm{e}^{\mathrm{i} S(r)},
$$

where the number of terms in the sum may vary with $r$. This expression raises a few delicate issues: First, how does one take into account the possibility of $\chi$ intersecting with part of the gauge orbits more than once? Second, does the orientation of the intersection play a role? Third, can I really ignore the sign of $\operatorname{det} K(A)$ in (3)?

An important insight was achieved by Gribov [2], who realized that, in Yang-Mills theory, where $A=A^{a} T^{a} \in s u(n)$, the Landau gauge $\chi=\partial \cdot A$ (like, in fact, any covariant gauge) is not ideal in the above sense, because a gauge orbit $\mathcal{O}_{A}$ may contain more than one configuration with $\chi=0$ :

$$
\exists A^{\prime}: \quad A^{\prime}=U(\mathrm{~d}+A) U^{\dagger} \quad \text { with } \quad \partial^{\mu} A_{\mu}=0=\partial^{\mu} A_{\mu}^{\prime} .
$$


If $A^{\prime}$ is infinitesimally close to $A$, one may approximate $U=\mathrm{e}^{\xi}=\mathrm{e}^{\xi^{a} T^{a}} \simeq \mathbb{1}+\xi$, and the existence of a so-called Gribov copy is equivalent to

$$
0=\partial^{\mu}\left(\partial_{\mu}+\operatorname{ad} A_{\mu}\right) \xi=\partial^{\mu} D_{\mu} \xi
$$

This condition on $A$ simply means that the Faddeev-Popov operator $K(A)=-\partial^{\mu} D_{\mu}$ (here in Landau gauge) possesses a non-constant zero mode. When $A$ is 'small', i.e. in perturbation theory, $K(A) \simeq-\partial^{\mu} \partial_{\mu}$ has only positive nontrivial eigenvalues, so no Gribov problem occurs. For the same reason, the problem does not appear in QED altogether. However, when $A$ becomes 'large enough', $K(A)$ eventually develops negative eigenvalues. At some critical 'size' of $A$, some eigenvalue of $K(A)$ crosses zero, meaning that $\operatorname{det} K(A)$ switches sign and a new Gribov copy will appear. Gribov [2] was the first to realize that many popular gauges (like Landau or Coulomb) yield infinitely many Gribov copies. Since then, such (infinitesimal) Gribov copies have been constructed rather explicitly.

It is customary to define the 'Gribov region' and 'first Gribov horizon' by

$$
\Omega:=\{A \mid \chi(A)=0 \& K(A)>0\} \quad \text { and } \quad \partial \Omega,
$$

respectively. Then, infinitesimal Gribov copies sit on either side of the Gribov horizon $\partial \Omega$. Let me list some important properties of the Gribov region, disregarding for this purpose global gauge transformations:

- An alternative definition is $\Omega=$ relative minima of $\|A\|^{2}=\operatorname{tr} \int \mathrm{d}^{d} x A \cdot A \quad$ on $\mathcal{O}_{A}$ \} because $\quad 0=\delta\|A\|^{2} \Leftrightarrow \partial \cdot A=0$ and $0<\delta^{2}\|A\|^{2} \Leftrightarrow-\partial \cdot D>0$.

- $\|A\|^{2}$ achieves its absolute minimum on each gauge orbit, thus each gauge orbit intersects $\Omega$.

- $\Omega$ is convex and bounded in every direction, because $\lim _{\lambda \rightarrow \infty} K(\lambda A)=\lambda \partial^{\mu} \operatorname{ad} A_{\mu} \quad$ is traceless. Hence, there exists a negative eigenvalue, so $\lambda A \notin \Omega$.

- The Gribov region still contains Gribov copies! The reason is that $\|A\|^{2}$ on $\mathcal{O}_{A}$ develops a saddle point at $\partial \Omega$. Therefore, $\|A\|^{2}$ can be lowered inside $\Omega$. Clearly, a gauge orbit $\mathcal{O}_{A}$ can feature more than one relative minimum of $\|A\|^{2}$.

In view of the above complications, a more strict notion is useful, and one defines the 'fundamental modular region' (FMR) as

$$
\Lambda:=\left\{\text { absolute minimum of }\|A\|^{2} \text { on } \mathcal{O}_{A}\right\}
$$

It follows that $\Lambda \subset \Omega, \mathcal{O} \cap \Lambda \neq 0, \Lambda$ is convex and bounded in all directions, and $\partial \Lambda \cap \partial \Omega \neq 0$. One should note, however, that degenerate absolute minima of $\|A\|^{2}$ live on $\partial \Lambda$, and so the boundary $\partial \Lambda$ still holds Gribov copies! Most of the historical material presented in this and the following section are taken from the review by Vandersickel and Zwanziger [1].

\section{Proposals for a remedy}

Over the years, a fair number of proposals have been put forward to control the Gribov copies. The ubiquity of the feature is epitomized by Singer's theorem [3], which states that only singular, i.e. non-continuous, gauges can be free of Gribov copies. 
An obvious reaction is to select such a singular gauge, for example a space-like planar or a hyperaxial one. Yet, these are very cumbersome, and the computational price may be too high.

It has been suggested to take (3) literally and to lift the absolute value of det $K$ into the action, but it remains unclear whether this procedure properly accounts for the number of Gribov copies. The opposite recipe maintains det $K$ without absolute value and integrates over all Gribov copies, hoping that alternating orientations of the intersection of $\mathcal{O}_{A}$ with the gauge condition will lead to a cancellation between most copies.

Other ideas invoke stochastic quantization, which introduces a 'gauge-fixing force' tangential to the gauge orbits, or simply a restriction of the functional integration to the Gribov region $\Omega$ or the FMR $\Lambda$. The latter is connected with a hope for a confinement mechanism: Since $\Omega$ is compact, quantization might give rise to a mass gap.

It is believed that the boundary of $\Omega$ carries a lot of weight, namely that the path integral is dominated by degenerate orbits

$$
\mathcal{O}_{\bar{A}} \text { for } \quad \bar{A} \text { with } D(\bar{A}) \xi=0 \quad \Longrightarrow \quad K(\bar{A})=0 \quad \Longrightarrow \quad \bar{A} \in \partial \Omega,
$$

so that $\operatorname{dim} \mathcal{O}_{\bar{A}}$ is smaller than the generic $\operatorname{dim} \mathcal{O}_{A}$ by the number of solutions $\xi$.

Denoting by $\kappa(A)$ the lowest eigenvalue of $K(A)$ and cutting off the functional integration at $\partial \Omega$, in a saddle-point approximation one obtains [1]

$$
\begin{aligned}
Z(J) & =\int \mathcal{D}(A, C, \bar{C}, B) \theta[\kappa(A)] \mathrm{e}^{\frac{\mathrm{i}}{\hbar}\left[S_{0}(A)+\int \bar{C} K C+\int \chi(A) B+\int J A\right]} \\
& =\frac{1}{2 \pi \mathrm{i}} \int_{-\infty}^{\infty} \frac{\mathrm{d} \omega}{\omega-\mathrm{i} \epsilon} \int \mathcal{D}(A, C, \bar{C}, B) \mathrm{e}^{\mathrm{i} \omega \kappa(A)} \mathrm{e}^{\frac{\mathrm{i}}{\hbar}\left[S_{0}(A)+\int \bar{C} K C+\int \chi(A) B+\int J A\right]} \\
& \stackrel{\text { saddle point }}{\simeq} \mathcal{D}(A, C, \bar{C}, B) \mathrm{e}^{\frac{\mathrm{i}}{\hbar}\left[S_{0}(A)+\int \bar{C} K C+\int \chi(A) B+\gamma^{2} H(A, C, \bar{C}, B)+\int J A\right]}
\end{aligned}
$$

with a 'horizon functional' $H$. In the Landau gauge, $\chi=\partial \cdot A$, the latter depends on $A$ only and has been computed to be [1]

$$
H(A)=\int \mathrm{d}^{d} x \int \mathrm{d}^{d} y f^{a b c} A_{\mu}^{b}(x)\left(K^{-1}\right)^{a d}(x-y) f^{d e c} A^{e \mu}(y)-\int \mathrm{d}^{d} x d\left(n^{2}-1\right),
$$

where $f^{a b c}$ denote the gauge-group structure constants. The 'Gribov parameter' $\gamma$ is to be determined self-consistently via the 'gap equation'

$$
\frac{\partial \ln Z(0)}{\partial \gamma}(\gamma)=0 \quad \Leftrightarrow \quad\langle H(A)\rangle_{\gamma}=0 \quad \text { 'horizon condition' . }
$$

Three remarks are in order: Firstly, the integration measure above peaks around $\partial \Omega$, supporting the 'degenerate-orbit dominance' hypothesis. Secondly, $\gamma \sim \exp \left\{-1 / g^{2}\right\}$ vanishes perturbatively, so its effect is only seen in the infrared. Thirdly, one finds that the gluon propagator behaves as $\frac{k^{2}}{k^{4}+2 \gamma^{2} g^{2} N}$ while the ghost propagator gets enhanced like $\frac{1}{k^{4}}$, consistent with the mass-gap picture.

\section{Yang-Mills theory in Faddeev-Popov quantization}

Almost all considerations regarding the Gribov problem have been made in the Landau gauge. However, for any proposal of overcoming the problem in a specific gauge, there arises the crucial issue of gauge invariance. It is therefore necessary to probe such proposals for nearby (or even 
distant) other gauges [4]. The proper tool for achieving this is a gauge-changing procedure for the generating functional, preferably in the BRST formulation.

I begin by reminding the audience of the salient features of the Faddeev-Popov quantization of $\mathrm{SU}(n)$ Yang-Mills theory in $\mathbb{R}^{1, d-1}$. Its classical action reads

$$
S_{0}(A)=-\frac{1}{4} \int \mathrm{d}^{d} x F_{\mu \nu}^{a} F^{\mu \nu a} \quad \text { with } \quad F_{\mu \nu}^{a}=\partial_{\mu} A_{\nu}^{a}-\partial_{\nu} A_{\mu}^{a}+f^{a b c} A_{\mu}^{b} A_{\nu}^{c},
$$

where $a=1, \ldots, n$ and $\mu=0,1, \ldots, d-1 . S_{0}$ is invariant under gauge transformations

$$
\delta A_{\mu}^{a}=D_{\mu}^{a b} \xi^{b} \quad \text { with } \quad D_{\mu}^{a b}=\delta^{a b} \partial_{\mu}+f^{a c b} A_{\mu}^{c} \quad \text { and } \quad \xi^{b}=\xi^{b}(x) .
$$

The BRST formulation of the quantum theory extends the field content and the action to

$$
\begin{gathered}
\left\{\phi^{A}\right\}=\left\{A_{\mu}^{a}(x), B^{a}(x), C^{a}(x), \bar{C}^{a}(x)\right\} \\
S(\phi)=S_{0}(A)+\int \mathrm{d}^{d} x \bar{C}^{a} K^{a b}(A) C^{b}+\int \mathrm{d}^{d} x \chi^{a}(A) B^{a},
\end{gathered}
$$

with a choice $\chi^{a}$ for a gauge-fixing function and with the ensuing Faddeev-Popov operator

$$
K^{a b}(A)=\frac{\partial \chi^{a}(A)}{\partial A_{\mu}^{c}} D_{\mu}^{c b} .
$$

In the Landau gauge, $\chi^{a}=\partial \cdot A^{a}$, one has $K^{a b}(A)=\delta^{a b} \partial^{\mu} \partial_{\mu}+f^{a c b} A_{\mu}^{c} \partial^{\mu}$.

The extended action $S$ is invariant under (even) BRST transformations

$$
\delta_{\lambda} A_{\mu}^{a}=D_{\mu}^{a b} C^{b} \lambda, \quad \delta_{\lambda} \bar{C}^{a}=B^{a} \lambda, \quad \delta_{\lambda} B^{a}=0, \quad \delta_{\lambda} C^{a}=\frac{1}{2} f^{a b c} C^{b} C^{c} \lambda,
$$

where $\lambda$ is an odd constant. It is convenient to introduce the (odd) Slavnov variation $s X$ of any functional $X$ by writing

$$
\delta_{\lambda} X(\phi)=(s X(\phi)) \lambda \quad \text { so that } \quad s X(\phi)=\frac{\delta X(\phi)}{\delta \phi^{A}} R^{A}(\phi),
$$

with the combined short-hand notation

$$
\left\{R^{A}(\phi)\right\}=\left\{D_{\mu}^{a b} C^{b}(x), 0, \frac{1}{2} f^{a b c} C^{b} C^{c}(x), B^{a}(x)\right\}
$$

and DeWitt's extension [5] of Einstein's summation convention (sum over $A$ includes integration over $x$ ). The nilpotency of the Slavnov variation, $s^{2}=0$, implies that

$$
0=s R^{A}(\phi)=\frac{\delta R^{A}(\phi)}{\delta \phi^{B}} R^{B}(\phi) \equiv R_{, B}^{A} R^{B} .
$$

It is very useful to define the extended (odd) gauge-fixing functional

$$
\psi(\phi)=\int \mathrm{d}^{d} x \bar{C}^{a} \chi^{a}(A)
$$

in terms of which the extended action can be made manifestly BRST invariant:

$$
S(\phi)=S_{0}(A)+\int \mathrm{d}^{d} x \bar{C}^{a} K^{a b}(A) C^{b}+\int \mathrm{d}^{d} x \chi^{a}(A) B^{a}=S_{0}(A)+s \psi(\phi)=: S_{\psi}(\phi),
$$

thus obviously $s S_{\psi}(\phi)=0$. 


\section{Field-dependent BRST transformations}

The main point of this talk, based on [6, 7], identifies a gauge change with a field-dependent BRST transformation. Let me therefore generalize the odd constant $\lambda$ to a field-dependent (but still $x$ independent) BRST-parameter functional $\Lambda(\phi)$. The corresponding transformation then reads

$$
\delta_{\Lambda} X(\phi)=(s X(\phi)) \Lambda(\phi)=X_{, A} R^{A} \Lambda(\phi) \quad \text { with } \quad \Lambda^{2}(\phi)=0 .
$$

On the fields $\phi^{A}$ themselves, this amounts to a (nonlocal) change of field variables $\phi \rightarrow \varphi$,

$$
\varphi^{A}=\varphi^{A}(\phi)=\phi^{A}+\delta_{\Lambda} \phi^{A}=\phi^{A}+\left(s \phi^{A}\right) \Lambda(\phi)=\phi^{A}+R^{A}(\phi) \Lambda(\phi),
$$

with a Jacobian supermatrix

$$
\begin{aligned}
M_{B}^{A}(\phi)=\frac{\delta \varphi^{A}(\phi)}{\delta \phi^{B}} & =\delta_{B}^{A}+\frac{\delta R^{A}(\phi)}{\delta \phi^{B}} \Lambda(\phi)(-1)^{\varepsilon_{B}}+R^{A}(\phi) \frac{\delta \Lambda(\phi)}{\delta \phi^{B}} \\
& \equiv \delta_{B}^{A}+R_{, B}^{A} \Lambda(-1)^{\varepsilon_{B}}+R^{A} \Lambda_{, B} .
\end{aligned}
$$

Surprisingly, its superdeterminant can be computed exactly:

$$
\begin{aligned}
\operatorname{sTr} \ln M(\phi) & =-\sum_{n=1}^{\infty} \frac{(-1)^{n}}{n} \operatorname{sTr}\left(R_{, B}^{A} \Lambda(-1)^{\varepsilon_{B}}+R^{A} \Lambda_{, B}\right)^{n} \\
& =-\sum_{n=1}^{\infty} \frac{(-1)^{n}}{n} \operatorname{sir}\left(R^{A} \Lambda_{, B}\right)^{n} \\
& =+\sum_{n=1}^{\infty} \frac{(-1)^{n}}{n}\left(\Lambda_{, A} R^{A}\right)^{n} \\
& =\sum_{n=1}^{\infty} \frac{(-1)^{n}}{n}(s \Lambda)^{n} \\
& =-\ln (1+s \Lambda(\phi)),
\end{aligned}
$$

hence

$$
\operatorname{sDet} M(\phi)=[1+s \Lambda(\phi)]^{-1} .
$$

Performing such a variable change in a functional integral, one obtains

$$
\begin{aligned}
\mathcal{I} & =\int \mathcal{D} \varphi \exp \left\{\frac{\mathrm{i}}{\hbar} W(\varphi)\right\} \\
& =\int \mathcal{D} \phi \operatorname{sDet} M(\phi) \exp \left\{\frac{\mathrm{i}}{\hbar} W(\varphi(\phi))\right\} \\
& =\int \mathcal{D} \phi \exp \left\{\frac{\mathrm{i}}{\hbar}[W(\varphi(\phi))-\mathrm{i} \hbar \mathrm{s} \operatorname{Tr} \ln M(\phi)]\right\} \\
& =\int \mathcal{D} \phi \exp \left\{\frac{\mathrm{i}}{\hbar}[W(\phi)+(s W(\phi)) \Lambda(\phi)+\mathrm{i} \hbar \ln (1+s \Lambda(\phi))]\right\},
\end{aligned}
$$

hence the functional $W$ is shifted by a classical and a quantum piece. It is important to realize that these transformations are not nilpotent, since

$$
\delta_{\Lambda}^{2} X(\phi)=\delta_{\Lambda}[(s X(\phi)) \Lambda(\phi)]=(s X(\phi))(s \Lambda(\phi)) \Lambda(\phi)
$$

vanishes only if

$$
0=s \Lambda(\phi)=\Lambda_{, A}(\phi) R^{A}(\phi),
$$

which of course includes the trivial case of $\Lambda(\phi)=\lambda=$ constant. 


\section{Relating different gauges}

I will now be more specific and take for $W$ the extended Yang-Mills action (24), i.e. investigate the form change of the Yang-Mills vacuum functional in a gauge $\psi$ under the change of field variables induced by a field-dependent BRST transformation:

$$
\begin{aligned}
Z_{\psi}(0) & =\int \mathcal{D} \varphi \exp \left\{\frac{\mathrm{i}}{\hbar} S_{\psi}(\varphi)\right\} \\
\stackrel{s S_{\psi}}{=}=0 & \int \mathcal{D} \phi \exp \left\{\frac{\mathrm{i}}{\hbar}\left[S_{\psi}(\phi)+\mathrm{i} \hbar \ln (1+s \Lambda(\phi))\right]\right\} \\
& =\int \mathcal{D} \phi \exp \left\{\frac{\mathrm{i}}{\hbar}\left[S_{0}(A)+s \psi(\phi)+s \delta \psi(\phi)\right]\right\}=Z_{\psi+\delta \psi}(0)
\end{aligned}
$$

where the last term in the exponent is BRST exact,

$$
\mathrm{i} \hbar \ln (1+s \Lambda(\phi))=s \delta \psi(\phi) \quad \text { with } \quad \delta \psi(\phi)=\mathrm{i} \hbar \Lambda(\phi)(s \Lambda(\phi))^{-1} \ln (1+s \Lambda(\phi)) .
$$

This shows that a field-dependent BRST transformation with $\Lambda$ effects a shift of the extended gauge-fixing functional by some $\delta \psi \simeq \mathrm{i} \hbar \Lambda+O\left(\Lambda^{2}\right)$.

It is illuminating to reverse the dependence and determine which BRST-parameter functional $\Lambda$ has to be chosen in order to achieve a given gauge change $\delta \psi$. The inversion

$$
s \Lambda(\phi)=\exp \left\{\frac{1}{\mathrm{i} \hbar} s \delta \psi\right\}-1
$$

is solved by (see also [8])

$$
\begin{aligned}
\Lambda(\phi) & =\delta \psi(s \delta \psi)^{-1}\left(\exp \left\{\frac{1}{\mathrm{i} \hbar} s \delta \psi\right\}-1\right) \\
& =\frac{1}{\mathrm{i} \hbar} \delta \psi \sum_{n=0}^{\infty} \frac{1}{(n+1) !}\left(\frac{s \delta \psi}{\mathrm{i} \hbar}\right)^{n} \simeq \frac{\delta \psi}{\mathrm{i} \hbar}+\frac{\delta \psi s \delta \psi}{2(\mathrm{i} \hbar)^{2}}+\ldots
\end{aligned}
$$

A prime example is the class of $R_{\xi}$ gauges, defined by

$$
\psi_{\xi}(\phi)=\int \mathrm{d}^{d} x \bar{C}^{a}\left(\partial^{\mu} A_{\mu}^{a}+\frac{\xi}{2} B^{a}\right) .
$$

To move from $R_{\xi}$ to $R_{\xi+\delta \xi}$ gauge needs

$$
\delta \psi=\frac{1}{2} \delta \xi \int \mathrm{d}^{d} x \bar{C}^{a} B^{a} \quad \Longrightarrow \quad s \delta \psi=\frac{1}{2} \delta \xi B^{2} \quad \text { with } \quad B^{2}=\int \mathrm{d}^{d} x B^{a} B^{a},
$$

and the corresponding field-dependent BRST-parameter functional reads

$$
\begin{aligned}
\Lambda(\phi) & =\left(B^{2}\right)^{-1}\left(\exp \left\{\frac{\delta \xi}{2 \mathrm{i} \hbar} B^{2}\right\}-1\right) \int \mathrm{d}^{d} x \bar{C}^{a} B^{a} \\
& =\frac{\delta \xi}{2 \mathrm{i} \hbar}\left\{1+\frac{1}{2 !} \frac{\delta \xi}{2 \mathrm{i} \hbar} B^{2}+\frac{1}{3 !}\left(\frac{\delta \xi}{2 \mathrm{i} \hbar} B^{2}\right)^{2}+\frac{1}{4 !}\left(\frac{\delta \xi}{2 \mathrm{i} \hbar} B^{2}\right)^{3}+\ldots\right\} \int \mathrm{d}^{d} x \bar{C}^{a} B^{a}
\end{aligned}
$$

in a (nonlocal) power series expansion in $\delta \xi\left(\right.$ and $\left.B^{2} / \hbar\right)$. 


\section{Gribov horizon beyond the Landau gauge}

The nonlocal Gribov-Zwanziger model [1] is defined by adding the (non-local) Gribov horizon functional to the extended action (24). In the Landau gauge, its (non-local) action then reads

$$
S_{H}(\phi)=S_{\psi}(\phi)+\gamma^{2} H_{0}(A)=S_{0}(A)+s \psi_{0}(\phi)+\gamma^{2} H_{0}(A)
$$

with

$$
\begin{aligned}
\psi_{0}(\phi) & =\int \mathrm{d}^{d} x \bar{C}^{a} \partial \cdot A^{a} \quad \text { and } \\
H_{0}(A) & =\int \mathrm{d}^{d} x \int \mathrm{d}^{d} y f^{a b c} A_{\mu}^{b}\left(K^{-1}\right)^{a d} f^{d e c} A^{e \mu}-\int \mathrm{d}^{d} x d\left(n^{2}-1\right),
\end{aligned}
$$

and the Gribov parameter $\gamma$ is determined self-consistently by the gap equation (13).

As it stands in (40), the Gribov-Zwanziger action $S_{H}$ is not BRST invariant, because $H$ is defined in the Landau gauge, and its Slavnov variation does not vanish,

$$
s H_{0}=\int \mathrm{d}^{d} x \int \mathrm{d}^{d} y f^{a b c} f^{c d e}\left[2 D_{\mu}^{b q} C^{q}\left(K^{-1}\right)^{a d}-f^{m p n} \int A_{\mu}^{b}\left(K^{-1}\right)^{a m} K^{p q} C^{q}\left(K^{-1}\right)^{n d}\right] A^{e \mu} \neq 0 .
$$

Does this imply that the effective quantum action and thus the physical S-matrix are gauge dependent even on-shell? No, it only means that away from the Landau gauge one cannot use the same horizon functional, but should properly modify it such as to account for the gauge change. This modification can now be directly domputed using the tool developed in the previous section.

For the example of the $R_{\xi}$ gauges (37), let me move from $\xi=0$ to some finite value of $\xi$. As I demonstrated, this can be done by a field-dependent BRST transformation,

$$
\phi^{A} \longmapsto \phi^{A}+\left(s \phi^{A}\right) \Lambda_{\xi}(\phi) \quad \text { with } \quad \Lambda_{\xi}(\phi)=\left(B^{2}\right)^{-1}\left(\exp \left\{\frac{\xi B^{2}}{2 \mathrm{i} \hbar}\right\}-1\right) \int \mathrm{d}^{d} x \bar{C}^{a} B^{a}
$$

leading to

$$
\begin{aligned}
Z_{\xi=0}(0) & =\int \mathcal{D} \phi \exp \left\{\frac{\mathrm{i}}{\hbar}\left(S_{0}(A)+s \psi_{0}(\phi)+\gamma^{2} H_{0}(A)\right)\right\} \\
& =\int \mathcal{D} \phi \exp \left\{\frac{\mathrm{i}}{\hbar}\left(S_{0}(A)+s \psi_{\xi}(\phi)+\gamma^{2} H_{\xi}(\phi)\right)\right\}=Z_{\xi}(0),
\end{aligned}
$$

where I read off

$$
\psi_{\xi}(\phi)=\int \mathrm{d}^{d} x \bar{C}^{a}\left(\partial^{\mu} A_{\mu}^{a}+\frac{\xi}{2} B^{a}\right) \quad \text { and } \quad H_{\xi}(\phi)=H_{0}(A)+\left(s H_{0}(A, C)\right) \Lambda_{\xi}(\phi) .
$$

The last equation provides a proposal for the horizon functional in a general $R_{\xi}$ gauge in such a way that gauge invariance is restored in the vacuum functional:

$$
\gamma^{2}\langle\delta H(\phi)\rangle_{\gamma}+\langle s \delta \psi(\phi)\rangle_{\gamma}=0 \quad \text { under } \quad \psi \mapsto \psi+\delta \psi
$$

To demonstrate the computability, here is its explicit form:

$$
\begin{aligned}
& H_{\xi}(\phi)=f^{a b c} \iint A_{\mu}^{b}\left(K^{-1}\right)^{a d} f^{d e c} A^{e \mu}-\int d\left(n^{2}-1\right) \\
& \quad+f^{a b c} f^{c d e} \iint\left[2 D_{\mu}^{b q} C^{q}\left(K^{-1}\right)^{a d}-f^{m p n} \int A_{\mu}^{b}\left(K^{-1}\right)^{a m} K^{p q} C^{q}\left(K^{-1}\right)^{n d}\right] A^{e \mu}\left(B^{2}\right)^{-1}\left(\mathrm{e}^{\frac{\xi}{2 \mathrm{i}}} B^{2}-1\right) \int \bar{C}^{\ell} B^{\ell} .
\end{aligned}
$$

This idea may likewise be applied to the local form of the Gribov-Zwanziger model [1], which further extends the field space to

$$
\left\{\Phi^{\mathcal{A}}\right\}=\left\{\phi^{A}, \varphi_{\mu}^{a c}, \bar{\varphi}_{\mu}^{a c}, \omega_{\mu}^{a c}, \bar{\omega}_{\mu}^{a c}\right\}
$$


and features the following (local) action,

$$
S_{G Z}(\Phi)=S_{0}(A)+s \psi(\phi)+S_{\gamma}(A, \varphi, \bar{\varphi}, \omega, \bar{\omega}),
$$

where the (now local) 'improvement functional' reads

$$
S_{\gamma}=\int \mathrm{d}^{d} x\left[\bar{\varphi}_{\mu}^{a c} K^{a b} \varphi^{\mu b c}-\bar{\omega}_{\mu}^{a c} K^{a b} \omega^{\mu b c}+2 \mathrm{i} \gamma f^{a b c} A_{\mu}^{b}\left(\varphi^{\mu a c}+\bar{\varphi}^{\mu a c}\right)+\gamma^{2} d\left(n^{2}-1\right)\right] .
$$

The new fields form two BRST doublets,

$$
\begin{array}{ll}
\delta_{\lambda} \varphi_{\mu}^{a c}=\omega_{\mu}^{a c} \lambda, & \delta_{\lambda} \bar{\varphi}_{\mu}^{a c}=0, \\
\delta_{\lambda} \omega_{\mu}^{a c}=0, & \delta_{\lambda} \bar{\omega}_{\mu}^{a c}=-\bar{\varphi}_{\mu}^{a c} \lambda .
\end{array}
$$

Again, BRST invariance is (softly) broken since

$s S_{\gamma}=f^{a d b} \int \mathrm{d}^{d} x\left[\bar{\varphi}_{\mu}^{a c} K^{d e} C^{e} \varphi^{\mu b c}+\bar{\omega}_{\mu}^{a c} K^{d e} C^{e} \omega^{\mu b c}+2 \mathrm{i} \gamma\left(D_{\mu}^{d e} C^{e}\left(\varphi^{\mu a b}+\bar{\varphi}^{\mu a b}\right)+A_{\mu}^{d} \omega^{\mu a b}\right)\right] \neq 0$.

However, gauge invariance can be restored by defining (for general $R_{\xi}$ gauges)

$$
S_{\gamma \xi}(\Phi)=S_{\gamma}(A, \varphi, \bar{\varphi}, \omega, \bar{\omega})+\left(s S_{\gamma}(A, C, \varphi, \bar{\varphi}, \omega, \bar{\omega})\right) \Lambda_{\xi}(\phi),
$$

so that the action changes via the corresponding field-dependent BRST transformation as

$$
S_{G Z}(\Phi) \quad \longmapsto \quad S_{0}(A)+s \psi_{\xi}(\phi)+S_{\gamma \xi}(\Phi)
$$

induced by a harmless change of variables in the functional integral. Once more,

$$
s S_{\gamma}(\Phi) \neq 0 \quad \text { but } \quad \delta S_{\gamma}(\Phi)=s S_{\gamma}(\Phi) \Lambda_{\xi}(\phi) \quad \text { balances } \quad s \delta \psi(\phi) .
$$

\section{Outlook}

I have shown how it is possible to move from a reference gauge $\psi_{0}$ to a desired gauge $\psi$ via a field-dependent BRST transformation with parameter functional

$$
\begin{aligned}
\Lambda_{\psi}(\phi) & =\left(\psi-\psi_{0}\right)\left(s\left(\psi-\psi_{0}\right)\right)^{-1}\left(\exp \left\{\frac{1}{\mathrm{i} \hbar} s\left(\psi-\psi_{0}\right)\right\}-1\right) \\
& =\frac{1}{\mathrm{i} \hbar}\left(\psi-\psi_{0}\right) \sum_{n=0}^{\infty} \frac{1}{(n+1) !}\left(\frac{1}{\mathrm{i} \hbar} s\left(\psi-\psi_{0}\right)\right)^{n} .
\end{aligned}
$$

This connection proposes a corresponding change of the horizon functional,

$$
H_{\psi}(\phi)-H_{0}(\phi)=\left(s H_{0}(\phi)\right) \Lambda_{\psi}(\phi) .
$$

One may use these results in $R_{\xi}$ gauges to interpolate between the interpretation-friendly unitary gauge $(\xi \rightarrow \infty)$ and the renormalization-friendly Landau gauge $(\xi \rightarrow 0)$. It will be also very interesting to go beyond $R_{\xi}$ gauges and relate, for instance, the Coulomb gauge to the Landau gauge. Finally, I have only discussed the vacuum functional. The analysis should be (and can be) extended to investigate the gauge variation of Greens functions, VEVs and S-matrix elements using

$$
Z_{\psi+\delta \psi}(J)=Z_{\psi}(J)-\frac{\mathrm{i}}{\hbar} J_{A}\left\langle\left(s \phi^{A}\right) \Lambda_{\delta \psi}(\phi)\right\rangle_{J} .
$$

\section{Acknowledgments}

I wish to thank Peter Lavrov for involving me into this project and for the pleasant collaboration. 


\section{References}

[1] N. Vandersickel and D. Zwanziger,

The Gribov problem and QCD dynamics,

Phys. Rept. 520 (2012) 175-251, arXiv: 1202 . 1491 [hep-th] .

[2] V.N. Gribov,

Quantization of nonabelian gauge theories,

Nucl. Phys. B 139 (1978) 1-19.

[3] I.M. Singer,

Some remarks on the Gribov ambiguity,

Commun. Math. Phys. 60 (1978) 7-12.

[4] P.M. Lavrov, O. Lechtenfeld and A.A. Reshetnyak, Is soft breaking of BRST symmetry consistent?,

JHEP 1110 (2011) 043, arXiv: 1108 . 4820 [hep-th] .

[5] B.S. DeWitt, Dynamical Theory of Groups and Fields, Gordon and Breach, New York, 1965.

[6] P.M. Lavrov and O. Lechtenfeld,

Field-dependent BRST transformations in Yang-Mills theory,

Phys. Lett. B 725 (2013) 382-385, arXiv:1305. 0712 [hep-th] .

[7] P.M. Lavrov and O. Lechtenfeld,

Gribov horizon beyond the Landau gauge,

Phys. Lett. B 725 (2013) 386-388, arXiv:1305.2931 [hep-th] .

[8] S.D. Joglekar and B.P. Mandal,

Finite field-dependent BRS transformations, Phys. Rev. D 51 (1995) 1919-1928. 